\title{
Mechanography-A New Device for the Assessment of Muscle Function in Pediatrics
}

\author{
OLIVER FRICKE, JUSTINE WEIDLER, BAERBEL TUTLEWSKI, AND ECKHARD SCHOENAU
}

Children's Hospital [O.F., J.W., B.T., E.S.], Department of Child and Adolescent Psychiatry [O.F.], University of Cologne, D-50924
Cologne, Germany

\begin{abstract}
The development of the musculoskeletal system in children and adolescents became an important topic in the field of pediatric research when the connections between muscle force and bone diseases were revealed. The present study focused on reference values of ground reaction forces, which derive from muscle forces of the lower limbs. Specifically, the study investigated the relationship between anthropometric characteristics and peak jump force (PJF), and peak jump power (PJP). The parameters were assessed by jumping mechanography using the Leonardo Jumping Platform. The entire study group was comprised of 135 school boys (ages 7-21 y) and 177 girls (ages 6-19 y), who were enrolled in a German primary school and a German high school. The analysis of the parameters revealed that forces deriving from the motor performance of jumping follow an exponential relationship to body size parameters. Therefore, with consideration of anthropometric characteristics, the assessment of ground reaction forces might provide a novel, inexpensive, and accurate approach for the assessment motor performance in children and adolescents. (Pediatr Res 59: 46-49, 2006)
\end{abstract}

$\mathrm{T}_{\mathrm{p}}$ he assessment of kinetic parameters deriving from motor performance has been recently recognized as an important issue for the investigation of biologic functions in humans (1). The dramatic change in the daily performance of motor activity in western industrial countries has resulted in an incredible increase in the prevalence of popular diseases in these societies. In highly developed countries, many individuals have replaced their own activity and motor performance with technical assistance in a computerized daily life. The combination of low motor activity with high caloric energy intake is one of the leading causes of prevalence of disease in industrial countries. Moreover, the associated increase of obesity in these societies increases the mortality in nonobesity-related critical health conditions (2). In addition, the decrease in daily motor activity of children changes the prevalence of several diseases [e.g. the number of children suffering from obesityrelated type 2 diabetes has increased in industrial countries the last years (3)]. This lack of motor activity in children can have dramatic immediate, as well as future, effects. Recent investigations have revealed the influence of muscular activity on bone mass as well as the mechanical properties of the skeleton in children and adolescents (4). The femoral neck is, besides

Received March 15, 2005; accepted July 5, 2005.

Correspondence: Oliver Fricke, M.D., Department of Child and Adolescent Psychiatry, Robert-Koch-Str. 10, D-50924 Cologne, Germany; e-mail: frickeo@ rockefeller.edu

DOI: 10.1203/01.pdr.0000191580.07644.1c the dorsal and lumbar spine, the most common location of osteoporotic fractures in elderly people. Therefore, the assessment of kinetic parameters, which are also related to motor performance of the lower limbs, might be a useful device for epidemiologic and individual investigations of the risk of osteoporosis. Recent studies have investigated parameters describing motor performance of the upper limbs (5). In contrast to grip strength, the motor action of the lower limbs is more complex, and, as a result, there are methodological difficulties in the evaluation of standardized data for distinct muscle forces. The present study evaluated motor performance of the lower limbs in young adolescents using jumping mechanography. If jumping mechanography is an accurate method for the assessing muscle function, it could be used to extend the already-known dependence between muscle force and bone mass in the forearm to the lower limbs.

In the present study, kinetic parameters are presented describing ground reaction forces in healthy children and adolescents. In addition, these data are used to calculate SD scores of the kinetic parameters. We present a new approach for the standardized assessment of muscle function in children and adolescents, which can be easily used to collect information about physical fitness. Therefore, jumping mechanography could be a novel and useful device for pediatricians.

\section{SUBJECTS AND METHODS}

Subjects. The measurements were performed on nonselected students (177 girls, 135 boys) at a German primary school and a German high-school. Therefore, individuals represent the average of the socioeconomic status of students attending a primary school or high school in the western part of Germany. Informed consent was obtained from all individuals and their parents. The study followed the guidelines of the review board of the University of Cologne.

Assessment of PJF and peak jump power PJP. Jumping forces were measured with the Leonardo Jumping Platform (Novotec GmbH, Pforzheim, Germany). This device measures forces applied to the plate over time. Therefore, stationary forces as well as the variation of forces over time (ground reaction forces) can be investigated (6). The platform is divided into two sections and can measure the applied forces from the right and left lower limb separately.

Principle of measurement. The applied force to the jumping platform is detected by four sensors on each part of the plate. The distribution of forces to the different sensors is dependent upon the area where the force is applied. 
The sum of all the forces of each plate, however, is not dependent on the location of application. Each sensor can detect a maximum of $900 \mathrm{~N}$.

The detection of force is performed by a deformation of the detector that is proportional to the applied force. The detector changes its electrical resistance proportionally to the deformation. Following Ohm's law, the voltage of the electric circuitry to which the detector is connected increases or decreases proportionally due to the applied force. This voltage change is increased by an amplifier, and detected and recorded by a personal computer.

The computer records the change of force measured over time. Therefore, kinetic parameters can be calculated when the body weight of the jumping individual is known. The software for the detection, storage and calculation of data were also manufactured by Novotec $\mathrm{GmbH}$

Performance of jumping. The individuals stood on the plate and each foot was placed on one section of the jumping platform. The jump was performed as a counter-movement jump with freely moving arms, and the subjects were instructed to jump as high as possible with the head and chest. Before the first jump, the weight force of the individual was measured to calculate body weight (weight force/acceleration of gravity $=$ body weight). The maximum of force of the ascending part of the jump was used for further calculations and is defined as PJF. The peak of the calculated power (force $\times$ velocity) is called PJP. The subjects performed three jumps and the highest peak of the three recordings was selected for further calculations. Subjects jumped using both feet and landed on both feet.

Assessment of parameters of the body size. Body weight was determined by use of the jumping plate as described above. Body height was determined using a stadiometer.

Statistical analysis. Descriptive statistics were calculated for the entire study population and are presented for sex, age, weight, and height. The parameters were transformed in logarithmic values that were used for further regression analysis. A linear regression analysis was performed for the prediction of PJF by MIGF. Moreover, $\mathrm{E}_{\mathrm{ST}}$ of PJF and PJP was calculated using data of six individuals, who performed the procedure of three jumps two times. The highest PJF and PJP of each performance were selected, and the $\mathrm{E}_{\mathrm{ST}}$ was calculated using the formula $\left[\left(\mathrm{X}_{1}-\mathrm{X}_{2}\right) \times 2\right] /\left(\mathrm{X}_{1}+\mathrm{X}_{2}\right)$, where $\mathrm{X}_{1}$ (test) and $X_{2}$ (retest) represent the highest value of each episode of three jumps. Furthermore, linear correlations between test and retest were performed. Statistical differences were considered significant at $p<0.05$. All statistical procedures were performed using PC Statistics 4.0 (Hoffmann Software, Gießen, Germany).

\section{RESULTS}

Anthropometric characteristics of the entire study population are presented in Table 1. The age range was $6-19$ y for girls and 7-21 y for boys. The relationship between muscular parameters and body size should be linear after logarithmization (5) and can be described as

Muscular parameter $=a$ [body size parameter $($ body mass) $]^{b}$. This logarithmic relationship was taken in account by the logarithmization of the data for body size (weight and height) and muscle performance (PJF, PJP). In boys, the

Table 1. Anthropometric characteristics of the entire study population

\begin{tabular}{ccc}
\hline & Mean \pm SD & Median $(\min -\max )$ \\
\hline Girls $(n=177)$ & & \\
Age $(\mathrm{y})$ & $11 \pm 3.4$ & $10(6-19)$ \\
Height $(\mathrm{cm})$ & $146.3 \pm 45.7$ & $145.6(113.2-179.9)$ \\
Weight $(\mathrm{kg})$ & $41.6 \pm 14.4$ & $39.7(18.8-93.3)$ \\
PJF $(\mathrm{N})$ & $1054 \pm 391$ & $1010(420-2400)$ \\
PJP (W) & $1646 \pm 732$ & $1410(580-3620)$ \\
MIGF (N) & $193 \pm 70$ & $177(59-402)$ \\
Boys $(n=135)$ & & \\
Age $(\mathrm{y})$ & $11 \pm 3.7$ & $10(7-21)$ \\
Height $(\mathrm{cm})$ & $151.0 \pm 19.2$ & $146.4(118.8-191.1)$ \\
Weight $(\mathrm{kg})$ & $45.6 \pm 17.7$ & $40.9(20.9-96.7)$ \\
PJF (N) & $1145 \pm 502$ & $1000(500-3030)$ \\
PJP (W) & $2024 \pm 1136$ & $1610(590-4910)$ \\
MIGF (N) & $240 \pm 120$ & $196(68-519)$ \\
\hline
\end{tabular}
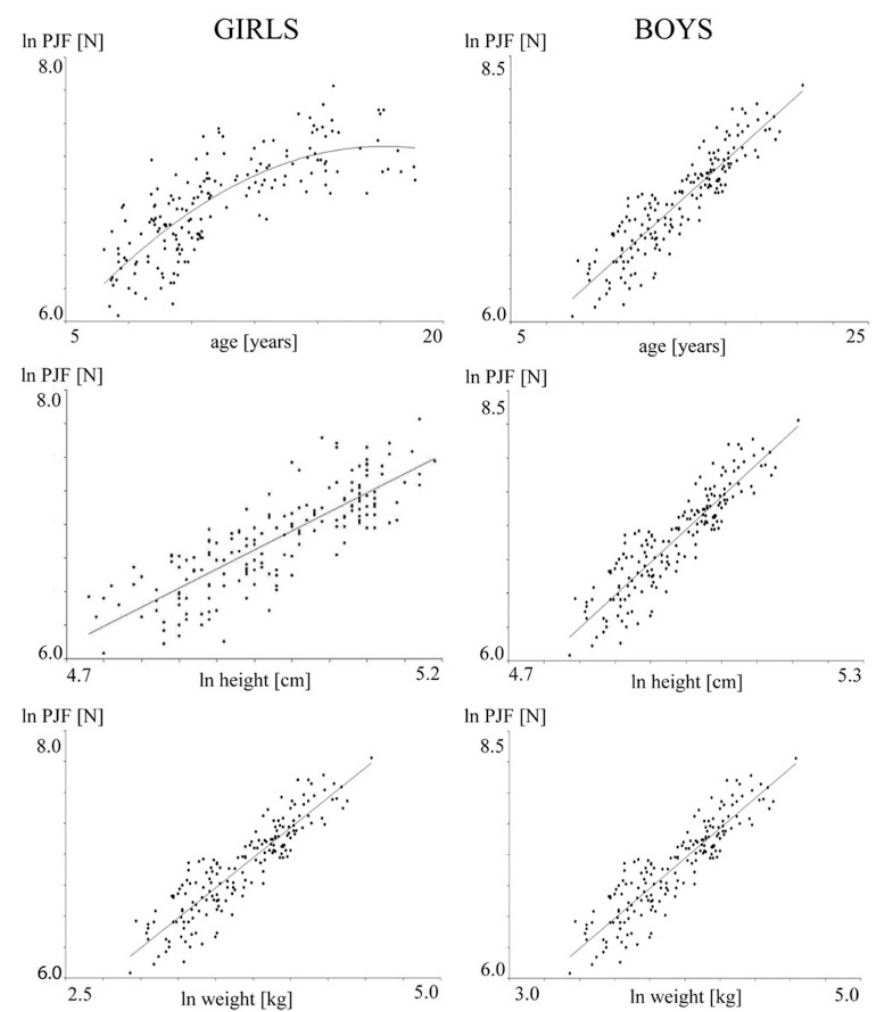

Figure 1. Regressions predicting $\ln (\mathrm{PJF})$. * Residual SD of the regression; $p$ $<0.01$ for all regression equations. Units: PJF, N; PJP, W; age, y; height, cm; weight, $\mathrm{kg}$.

age-related increase of PJF and PJP follows a linear association to $\ln (\mathrm{PJF})$ and $\ln (\mathrm{PJP})$. In girls, however, this relationship is better described by a polynomial of the second order. The relationship between body size (weight and height) follows the logarithmic transformation of PJF and PJP in a linear fashion (Figs. 1 and 2; Tables 2-4 show reference data for PJF and PJP in relation to a stepwise increase of age, height, and weight.

To observe a difference between genders, the point of intersection of the lines indicating the $+2 \mathrm{SD}$ range of the regression line with the lower constant and the -2 SD range of the regression line with the higher constant were calculated for $\ln$ (weight) and ln (height), predicting $\ln$ (PJP). If the abscissa of the point of intersection is lower than the lowest value of weight or height in the study group, more than $5 \%$ of the subjects will fall within the intersection area bound by the ranges $(-2 \mathrm{SD}$ to $+2 \mathrm{SD})$ of both genders. When muscular parameters were predicted by the regression lines, the difference was not significant. The point of intersection of lines indicating ranges for $\ln$ (weight) was determined to be -36.46 $+\ln (\mathrm{kg})$; predicting $\ln (\mathrm{PJP})$, the point of intersection of lines indicating the ranges of $\ln$ (height) was calculated to be $2.7+$ $\ln (\mathrm{cm})$. Both values are out of the range of weight and height of the study group. Therefore, the models predicting PJP by weight and height are not significantly different between males and females in the study group. The same procedure was performed for the prediction of ln (PJF). The calculated abscissa values of points of intersection of the gender-related regression lines are also lower as the lowest measured value of weight and height of the entire study group (weight: point of 

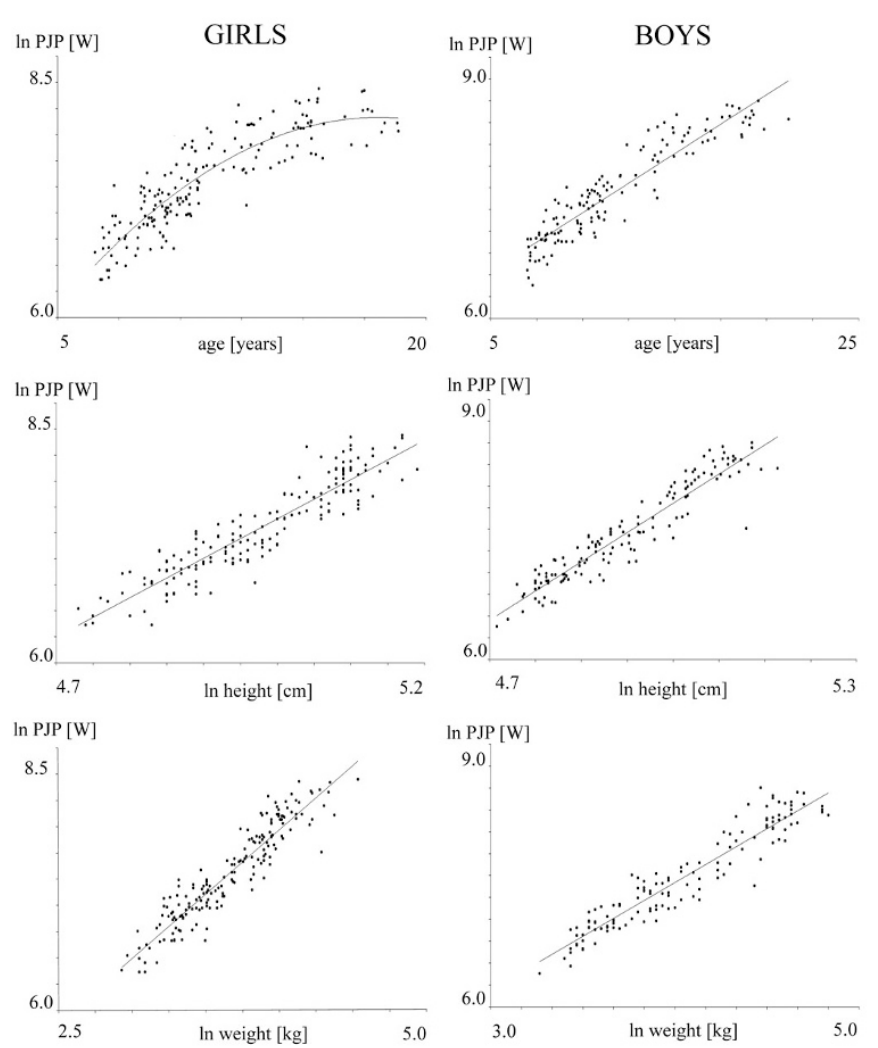

Figure 2. Regressions predicting $\ln$ (PJP). *Residual SD of the regression; $p$ $<0.01$ for all regression equations. Units: PJF, N; PJP, W; age, y; height, cm; weight, $\mathrm{kg}$.

Table 2. Reference values for PJF $(N)$ and $P J P(W)$ by age

\begin{tabular}{|c|c|c|c|c|}
\hline \multirow{3}{*}{$\begin{array}{l}\text { Age } \\
(\mathrm{yr})\end{array}$} & \multicolumn{2}{|c|}{ Mean \pm SD for PJF $(\mathrm{N})$} & \multicolumn{2}{|c|}{ Mean \pm SD for PJP $(\mathrm{W})$} \\
\hline & \multirow{2}{*}{$\begin{array}{c}\text { Girls } \\
(n=177)\end{array}$} & & & \\
\hline & & $\begin{array}{c}\text { Boys } \\
(n=135)\end{array}$ & Girls & Boys \\
\hline 6 & $597 \pm 126(6)$ & & $750 \pm 136$ & \\
\hline 7 & $660 \pm 149(17)$ & $677 \pm 119(20)$ & $853 \pm 204$ & $895 \pm 159$ \\
\hline 8 & $807 \pm 198(25)$ & $723 \pm 115(18)$ & $1092 \pm 220$ & $1116 \pm 218$ \\
\hline 9 & $793 \pm 187(31)$ & $799 \pm 190(15)$ & $1171 \pm 230$ & $1300 \pm 251$ \\
\hline 10 & $957 \pm 296(15)$ & $970 \pm 172(21)$ & $1409 \pm 352$ & $1510 \pm 258$ \\
\hline 11 & $1130 \pm 210(14)$ & $1206 \pm 414(8)$ & $1629 \pm 305$ & $1697 \pm 282$ \\
\hline 12 & $1266 \pm 294(8)$ & $1120 \pm 233(7)$ & $1944 \pm 417$ & $1996 \pm 513$ \\
\hline 13 & $1217 \pm 190(13)$ & $1470 \pm 235(8)$ & $2157 \pm 479$ & $2781 \pm 722$ \\
\hline 14 & $1298 \pm 160(9)$ & $1601 \pm 393(10)$ & $2268 \pm 400$ & $2986 \pm 675$ \\
\hline 15 & $1493 \pm 292(11)$ & $1660 \pm 248(6)$ & $2358 \pm 495$ & $3389 \pm 512$ \\
\hline 16 & $1559 \pm 363(16)$ & $1627 \pm 406(6)$ & $2638 \pm 507$ & $3298 \pm 454$ \\
\hline 17 & $1275 \pm 304(2)$ & $1718 \pm 413(3)$ & $2425 \pm 460$ & $3980 \pm 753$ \\
\hline 18 & $1675 \pm 352(6)$ & $1923 \pm 121(7)$ & $2978 \pm 528$ & $3950 \pm 532$ \\
\hline$>19$ & $1300 \pm 123(4)$ & $2018 \pm 564(6)$ & $2482 \pm 119$ & $4123 \pm 486$ \\
\hline
\end{tabular}

The number of individuals is presented in parentheses and is not different between PJF and PJP.

intersection at $0.083+\ln (\mathrm{kg})$; height: point of intersection at $3.36+\ln (\mathrm{cm}))$. Therefore, $\ln (\mathrm{PJF})$ predicted by the linear regression lines is also not significantly different between genders in the entire study group.

The mean of $\mathrm{E}_{\mathrm{ST}}$ was $3.69 \pm 0.95 \%$ (range, $0.85-8.23 \%$ ) for PJP, and $8.88 \pm 2.26 \%$ for PJF (range, $1.36-30.76 \%$ ). The correlation coefficients of the relation between test and retest were calculated to be $r=0.98$ for PJP, and $r=0.93$ for $\mathrm{PJF}$ (regression equations were significant, with $p<0.05$ for each).
Table 3. Reference values for PJF $(N)$ and $P J P(W)$ by height

\begin{tabular}{cccccc}
\hline & \multicolumn{2}{c}{ Mean \pm SD for PJF $(\mathrm{N})$} & & \multicolumn{2}{c}{ Mean \pm SD for PJP $(\mathrm{W})$} \\
\cline { 2 - 3 } $\begin{array}{c}\text { Height } \\
(\mathrm{cm})\end{array}$ & $\begin{array}{c}\text { Girls } \\
(n=177)\end{array}$ & $\begin{array}{c}\text { Boys } \\
(n=132)\end{array}$ & & Girls & Boys \\
\hline 110 & $588 \pm 95(6)$ & & $660 \pm 72$ & \\
120 & $660 \pm 128(27)$ & $669 \pm 102(22)$ & $897 \pm 158$ & $913 \pm 151$ \\
130 & $793 \pm 161(35)$ & $739 \pm 132(23)$ & $1131 \pm 263$ & $1131 \pm 258$ \\
140 & $984 \pm 252(37)$ & $928 \pm 170(29)$ & & $1392 \pm 263$ & $1484 \pm 231$ \\
150 & $1289 \pm 324(22)$ & $1183 \pm 277(13)$ & $2031 \pm 410$ & $1845 \pm 327$ \\
160 & $1420 \pm 243(42)$ & $1413 \pm 251(12)$ & $2482 \pm 417$ & $2692 \pm 691$ \\
170 & $1625 \pm 394(8)$ & $1800 \pm 399(26)$ & $2868 \pm 487$ & $3513 \pm 571$ \\
180 & & $1874 \pm 151(7)$ & & $4031 \pm 492$ \\
\hline
\end{tabular}

The number of individuals is presented in parentheses and is not different between PJF and PJP.

Table 4. Reference values for PJF $(N)$ and $P J P(W)$ by weight

\begin{tabular}{cccccc}
\hline & \multicolumn{2}{c}{ Mean \pm SD for PJF $(\mathrm{N})$} & & \\
\cline { 2 - 3 } \cline { 5 - 6 } $\begin{array}{c}\text { Weight } \\
(\mathrm{kg})\end{array}$ & $\begin{array}{c}\text { Girls } \\
(n=174)\end{array}$ & $\begin{array}{c}\text { Boys } \\
(n=133)\end{array}$ & Gean $\pm \mathrm{SD}$ for PJP $(\mathrm{W})$ \\
\hline 20 & $576 \pm 90(13)$ & $616 \pm 130(6)$ & $745 \pm 131$ & $760 \pm 139$ \\
25 & $719 \pm 143(32)$ & $665 \pm 82(25)$ & $1002 \pm 156$ & $986 \pm 147$ \\
30 & $821 \pm 146(28)$ & $774 \pm 118(19)$ & $1164 \pm 175$ & $1226 \pm 214$ \\
35 & $876 \pm 116(14)$ & $915 \pm 115(17)$ & $1307 \pm 186$ & $1431 \pm 224$ \\
40 & $1086 \pm 142(14)$ & $1028 \pm 144(10)$ & $1739 \pm 279$ & $1567 \pm 247$ \\
45 & $1206 \pm 162(22)$ & $1147 \pm 128(9)$ & $1864 \pm 350$ & $1918 \pm 262$ \\
50 & $1273 \pm 176(22)$ & $1290 \pm 122(7)$ & $2288 \pm 409$ & $2420 \pm 480$ \\
55 & $1535 \pm 212(13)$ & $1454 \pm 219(11)$ & $2518 \pm 303$ & $2724 \pm 472$ \\
60 & $1717 \pm 212(6)$ & $1650 \pm 299(9)$ & $2839 \pm 385$ & $3494 \pm 402$ \\
65 & $1697 \pm 331(4)$ & $1838 \pm 126(5)$ & $2800 \pm 465$ & $3758 \pm 498$ \\
70 & $1790 \pm 130(3)$ & $2021 \pm 116(7)$ & $2617 \pm 742$ & $3973 \pm 488$ \\
75 & $1717 \pm 148(3)$ & $1902 \pm 117(4)$ & $309 \pm 481$ & $3937 \pm 304$ \\
80 & & $2150 \pm 609(4)$ & & $3795 \pm 1390$
\end{tabular}

The number of individuals is presented in parentheses and is not different between PJF and PJP.

\section{DISCUSSION}

The present study provides information on parameters describing muscle function involved in the motor performance of jumping, called ground reaction forces (6-8). The performed jump was less standardized in an anatomical manner as more characterized by the demand to jump as high as possible in a counter-movement jump. Therefore, the assessed parameters PJF and PJP reflect the output of the complete motor system and are not derived from distinct anatomical areas under special mechanical conditions. Moreover, this test does not give a distinct view on parameters of muscle function (i.e. velocity of contraction or muscular coordination). More likely, the parameters PJF and PJP provide information on the daily performance of the motor system presenting an array of muscle coordination, structural and metabolic characteristics of the muscle system. Rittweger et al. (6) recently described the good reproducibility of PJP in elderly people with a small $\mathrm{E}_{\mathrm{ST}}(3.6 \%)$ and a high test-retest correlation coefficient $\left(r^{2}=\right.$ 0.98), which is in concordance with the results of the present study $\left(\mathrm{E}_{\mathrm{ST}}=3.69 \%, r^{2}=0.96\right)$. Long time reliability is a problematic issue in children and adolescents, because they change body size (weight and height) over time. This increase depends on growth and might be several centimeters over 3-6 months in puberty. Because muscle function depends on 
auxological characteristics, measurements of long time reliability will be remarkably influenced by growth. Therefore, long time reliability seems not to be a useful parameter of test reliability of muscle function in children and adolescents

Study reference data are presented in three tables (Tables 2-4) reflecting PJF and PJP in relationship to age and parameters of body size. These data provide additional information to recently published data on muscle force in children and adolescents (5,9-12). In addition, the present results are the first data to measure the motor performance of jumping in a larger group of children and adolescents using a new device of (jumping mechanography) which accurately assesses ground reaction forces.

The prediction of $\ln (\mathrm{PJF})$ and $\ln (\mathrm{PJP})$ by weight and height did not reveal a difference between genders. However, it is important to keep in mind that the prediction model is calculated over the complete range of age and, therefore, comprises prepubertal and postpubertal individuals. If differences between prepubertal and postpubertal individuals exist, they would not have been revealed by this design of analysis. Sex-related differences are described in recently published data that emphasize the differences in musculoskeletal development between genders (4). Males develop higher MGF than females starting at puberty, whereas the female skeleton builds up more bone mass in relation to mechanical forces than the male skeleton. This difference can be explained by genderrelated differences in steroid hormones. Androgenic hormones increase muscle mass, whereas estrogens increase the gain of bone mass due to applied mechanical forces

In conclusion, the present study presents reference values of ground reaction forces measured by jumping mechanography in children and adolescents. The establishment of reference values for PJF and PJP will be a useful and inexpensive device for assessing of the motor system in children and adolescents. The present data might serve to inform scientists and pediatricians about the relationship between body size and ground reaction forces in children and adolescents.

Acknowledgments. The authors thank Dr. Aaron Jasnow for his helpful advice in the preparation of the manuscript

\section{REFERENCES}

1. Frost HM, Schonau E 2000 The "muscle-bone unit" in children and adolescents: a 2000 overview. J Pediatr Endocrinol Metab 13:571-590

2. Bercault N, Boulain T, Kuteifan K, Wolf M, Runge I, Fleury JC 2004 Obesityrelated excess mortality rate in an adult intensive care unit: a risk-adjusted matched cohort study. Crit Care Med 32:998-1003

3. Ramchandani N 2004 Type 2 diabetes in children. A burgeoning health problem among overweight young Americans. Am J Nurs 104:65-68

4. Schoenau E, Neu CM, Mokov E, Wassmer G, Manz F 2000 Influence of puberty on muscle area and cortical bone area of the forearm in boys and girls. J Clin Endocrinol Metab 85:1095-1098

5. Rauch F, Neu CM, Wassmer G, Beck B, Rieger-Wettengl G, Rietschel E, Manz F, Schoenau E 2002 Muscle analysis by measurement of maximal isometric grip force: new reference data and clinical applications in pediatrics. Pediatr Res 51:505-510

6. Rittweger J, Schiessl H, Felsenberg D, Runge M 2004 Reproducibility of the jumping mechanography as a test of mechanical power output in physically competent adult and elderly subjects. J Am Geriatr Soc 52:128-131

7. Cavagna GA, Komarek L, Mazzoleni S 1971 The mechanics of sprint running. J Physiol 217:709-721

8. Cavagna GA 1975 Force platforms as ergometers. J Appl Physiol 39:174-179

9. Mathiowetz V, Wiemer DM, Federman SM 1986 Grip and pinch strength: norms for 6-19 years-old. Am J Occup Ther 40:705-711

10. Sinaki M, Limburg PJ, Wollan PC, Rogers JW, Murtaugh PA 1996 Correlation of trunk muscle strength with age in children 5 to 18 years old. Mayo Clin Proc 71:1047-1054

11. Parker DF, Round JM, Sacco P, Jones DA 1990 A cross-sectional survey of upper and lower limb strength in boys and girls during childhood and adolescents. Ann Hum Biol 17:199-211

12. Round JM, Jones DA, Honour JW, Nevill AM 1999 Hormonal factors in the development of differences in strength between boys and girls during adolescence: a longitudinal study. Ann Hum Biol 26:49-62 